\title{
Hajós and Ore constructions for digraphs
}

\author{
Jørgen Bang-Jensen* Thomas Bellitto* \\ IMADA \\ University of Southern Denmark \\ Campusvej 55, DK-5320 Odense M, Denmark \\ $\{j b j$, bellitto\}@imada.sdu.dk \\ Thomas Schweser* Michael Stiebitz* \\ Institute of Mathematics \\ Technische Universität Ilmenau \\ D-98684 Ilmenau, Germany \\ \{thomas.schweser, michael.stiebitz\}@tu-ilmenau.de
}

Submitted: Aug 12, 2019; Accepted: Mar 2, 2020; Published: Mar 20, 2020

(c) The authors. Released under the CC BY-ND license (International 4.0).

\begin{abstract}
The dichromatic number $\vec{\chi}(D)$ of a digraph $D$ is the minimum number of colors needed to color the vertices of $D$ such that each color class induces an acyclic subdigraph of $D$. A digraph $D$ is $k$-critical if $\vec{\chi}(D)=k$ but $\vec{\chi}\left(D^{\prime}\right)<k$ for all proper subdigraphs $D^{\prime}$ of $D$. We examine methods for creating infinite families of critical digraphs, the Dirac join and the directed and bidirected Hajós join. We prove that a digraph $D$ has dichromatic number at least $k$ if and only if it contains a subdigraph that can be obtained from bidirected complete graphs on $k$ vertices by directed Hajós joins and identifying non-adjacent vertices. Building upon that, we show that a digraph $D$ has dichromatic number at least $k$ if and only if it can be constructed from bidirected $K_{k}$ 's by using directed and bidirected Hajós joins and identifying non-adjacent vertices (so called Ore joins), thereby transferring a well-known result of Urquhart to digraphs. Finally, we prove a Gallai-type theorem that characterizes the structure of the low vertex subdigraph of a critical digraph, that is, the subdigraph, which is induced by the vertices that have in-degree $k-1$ and out-degree $k-1$ in $D$.
\end{abstract}

Mathematics Subject Classifications: 05C20

${ }^{*}$ Research supported by the Independent Research Fond Denmark under grant number DFF 701400037B 


\section{Introduction}

Recall that the chromatic number $\chi(G)$ of a graph $G$ is the minimum number of colors needed to color the vertices of $G$ so that each color class induces an edgeless subgraph of $G$. The term graph refers to a finite, undirected, and simple graph. A graph $G$ is $k$-critical if $\chi(G)=k$ but $\chi\left(G^{\prime}\right)<k$ for each proper subgraph $G^{\prime}$ of $G$. The topic of critical graphs has received much attention within the last seven decades. Critical graphs were first introduced by Dirac in his doctoral thesis [5]; famous mathematicians like Hajós, Gallai, and others continued developing the theory of critical graphs in the 1960's (see, for instance, $[7,8,10,29])$.

However, there is not much known regarding critical digraphs. Following NeumannLara [27], a $k$-coloring of a digraph $D$ is a mapping $\varphi: V(D) \rightarrow\{1,2, \ldots, k\}$, such that for each color $\alpha \in\{1,2, \ldots, k\}$ the color class $\varphi^{-1}(\alpha)=\{v \in V(D) \mid \varphi(v)=\alpha\}$ induces an acyclic subdigraph of $D$, i.e. a subdigraph that does not contain any directed cycles. The dichromatic number $\vec{\chi}(D)$ of a digraph $D$ is the minimum integer $k \geqslant 0$ such that $D$ admits a $k$-coloring. A digraph $D$ is critical and $k$-critical if $\vec{\chi}(D)=k$ but $\vec{\chi}\left(D^{\prime}\right)<k$ for each proper subdigraph of $D$. Let $\operatorname{Crit}(k)$ denote the class of $k$-critical digraphs. Then, it is easy to see that $\operatorname{Crit}(0)=\{\varnothing\}$, Crit $(1)=\left\{K_{1}\right\}$, and $\operatorname{Crit}(2)$ consists of all directed cycles. Nevertheless, it is not even known which digraphs Crit(3) consists of; unlike in the undirected case, where it follows from König's characterization of bipartite graphs [19] that Crit(3) coincides with the class of all odd cycles. In this paper, we study the digraph analogue of two well-known methods for creating infinite families of critical graphs, the so-called Dirac join and the directed and bidirected Hajós join. Moreover, we prove that a digraph $D$ has dichromatic number at least $k$ if and only if it contains a Hajós- $k$ constructible subdigraph, that is, a subdigraph of $D$ that can be obtained from bidirected $K_{k}$ 's by iteratively applying the directed Hajós join and identifying non-adjacent vertices (see Theorem 4). In Section 5 we prove a Gallai-type theorem that characterizes the structure of the low-vertex subdigraph of a $k$-critical digraph, that is, the subdigraph that is induced by the vertices having in-degree and out-degree $k-1$ (see Theorem 15).

\section{Basic Terminology}

Most of our terminology is defined as in [2]. Let $D=(V(D), A(D))$ be a digraph. Then, $V(D)$ is the set of vertices of $D$ and $A(D)$ is the set of arcs of $D$. The order $|D|$ of $D$ is the size of $V(D)$. Digraphs in this paper are not allowed to have loops nor parallel arcs; however, there may be two arcs in opposite directions between two vertices (in this case we say that the arcs are opposite). We denote by $u v$ the arc whose initial vertex is $u$ and whose terminal vertex is $v$. Two vertices $u, v$ are adjacent if at least one of $u v$ and $v u$ belongs to $A(D)$. If $u$ and $v$ are adjacent, we also say that $u$ is a neighbor of $v$ and vice versa. If $u v \in A(D)$, then $v$ is called an out-neighbor of $u$ and $u$ is called an in-neighbor of $v$. By $N_{D}^{+}(v)$ we denote the set of out-neighbors of $v$; by $N_{D}^{-}(v)$ the set of in-neighbors of $v$. The out-degree of a vertex $v \in V(D)$ is the number of arcs whose inital vertex is $v$; we denote it by $d_{D}^{+}(v)$. Similarly, the number of arcs whose 
terminal vertex is $v$ is called the in-degree of $v$ and is denoted by $d_{D}^{-}(v)$. Note that $d_{D}^{+}(v)=\left|N_{D}^{+}(v)\right|$ and $d_{D}^{-}(v)=\left|N_{D}^{-}(v)\right|$ for all $v \in V(D)$. A vertex $v \in V(D)$ is Eulerian if $d_{D}^{+}(v)=d_{D}^{-}(v)$. Moreover, the digraph $D$ is Eulerian if every vertex of $D$ is Eulerian. Given a vertex set $X \subseteq V(D)$, by $D[X]$ we denote the subdigraph of $D$ that is induced by the vertex set $X$, that is, $V(D[X])=X$ and $A(D[X])=\{u v \in A(D) \mid u, v \in X\}$. A digraph $D^{\prime}$ is said to be an induced subdigraph of $D$ if $D^{\prime}=D\left[V\left(D^{\prime}\right)\right]$. As usual, if $X$ is a subset of $V(D)$, we define $D-X=D[V(D) \backslash X]$. If $X=\{v\}$ is a singleton, we use $D-v$ rather than $D-\{v\}$. Given a digraph $D$, its complement is the digraph $\bar{D}$ with $V(\bar{D})=V(D)$ and

$$
A(\bar{D})=\{u v \mid u, v \in V(D) \text { and } u v \notin A(D)\}
$$

The underlying (simple) graph $G(D)$ of $D$ is the graph with $V(G(D))=V(D)$ and $\{u, v\} \in E(G(D))$ if and only if at least one of $u v$ and $v u$ belongs to $A(D)$. The digraph $D$ is (weakly) connected if $G(D)$ is connected. A separating vertex of a connected digraph $D$ is a vertex $v \in V(D)$ such that $D-v$ is not connected. Furthermore, a block of $D$ is a maximal connected subdigraph $D^{\prime}$ of $D$ such that $D^{\prime}$ has no separating vertex.

A directed path of length $p-1 \geqslant 0$ is a digraph $P$ with $V(P)=\left\{v_{1}, v_{2}, \ldots, v_{p}\right\}$ and $A(P)=\left\{v_{1} v_{2}, v_{2} v_{3}, \ldots, v_{p-1} v_{p}\right\}$ where the $v_{i}$ are all distinct. Furthermore, a directed cycle of length $p \geqslant 2$ is a digraph $C$ with $V(C)=\left\{v_{1}, v_{2}, \ldots, v_{p}\right\}$ and $A(C)=$ $\left\{v_{1} v_{2}, v_{2} v_{3}, \ldots, v_{p-1} v_{p}, v_{p} v_{1}\right\}$ where the $v_{i}$ are all distinct. A directed cycle of length 2 is called a digon. A bidirected graph is a digraph that can be obtained from a graph $G$ by replacing each edge by two opposite arcs, we denote it by $D(G)$. A bidirected complete graph is also called a complete digraph. It is easy to see that $\vec{\chi}(D(G))=\chi(G)$ and $D(G)$ is critical with respect to $\vec{\chi}$ if and only if $G$ is critical with respect to $\chi$.

\section{Construction of critical digraphs}

Let $D_{1}$ and $D_{2}$ be two disjoint digraphs. Let $D$ be the digraph obtained from the union $D_{1} \cup D_{2}$ by adding all possible arcs in both directions between $D_{1}$ and $D_{2}$, i.e., $V(D)=$ $V\left(D_{1}\right) \cup V\left(D_{2}\right)$ and $A(D)=A\left(D_{1}\right) \cup A\left(D_{2}\right) \cup\left\{u v, v u \mid u \in V\left(D_{1}\right)\right.$ and $\left.v \in V\left(D_{2}\right)\right\}$. We say that $D$ is the Dirac join of $D_{1}$ and $D_{2}$ and denote it by $D=D_{1} \boxplus D_{2}$. The proof of the next theorem is straightforward and therefore left to the reader.

Theorem 1 (Dirac Construction). Let $D=D_{1} \boxplus D_{2}$ be the Dirac join of two disjoint non-empty digraphs $D_{1}$ and $D_{2}$. Then, $\vec{\chi}(D)=\vec{\chi}\left(D_{1}\right)+\vec{\chi}\left(D_{2}\right)$ and $D$ is critical if and only if both $D_{1}$ and $D_{2}$ are critical.

The Hajós join is a well-known tool for undirected graphs that can be used to create infinite families of $k$-critical graphs, see e. g. [10]. For digraphs, an equivalent construction was defined by Hoshino and Kawarabayashi in [15]. Let $D_{1}$ and $D_{2}$ be two disjoint digraphs and select an $\operatorname{arc} u_{1} v_{1}$ of $D_{1}$ as well as an arc $v_{2} u_{2}$ of $D_{2}$. Let $D$ be the digraph obtained from the union $D_{1} \cup D_{2}$ by deleting both arcs $u_{1} v_{1}$ and $v_{2} u_{2}$, identifying the vertices $v_{1}$ and $v_{2}$ to a new vertex $v$, and adding the $\operatorname{arc} u_{1} u_{2}$. We say that $D$ is the 
(directed) Hajós join of $D_{1}$ and $D_{2}$ and write $D=\left(D_{1}, v_{1}, u_{1}\right) \nabla\left(D_{2}, v_{2}, u_{2}\right)$ or, briefly, $D=D_{1} \nabla D_{2}$. Note that for the undirected Hajós join of two undirected graphs $G_{1}$ and $G_{2}$, we just choose two edges $u_{1} v_{1} \in E\left(G_{1}\right)$ and $u_{2} v_{2} \in E\left(G_{2}\right)$ and perform exactly the same procedure as described above (except for the orientations). Statement (c) of the following theorem has already been mentioned in [15, Proposition 2].
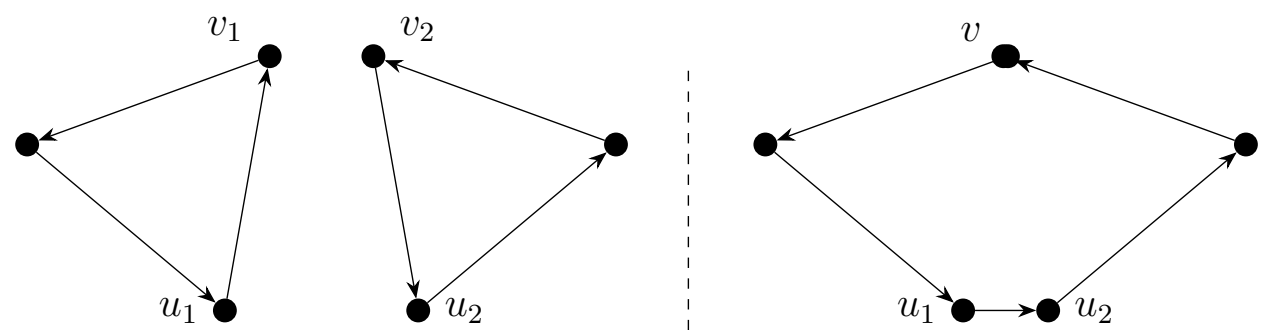

Figure 1: The Hajós join of two directed cycles of length 3

Theorem 2 (Hajós Construction). Let $D=D_{1} \nabla D_{2}$ be the Hajós join of two disjoint non-empty digraphs $D_{1}$ and $D_{2}$. Then, the following statements hold:

(a) $\vec{\chi}(D) \geqslant \min \left\{\vec{\chi}\left(D_{1}\right), \vec{\chi}\left(D_{2}\right)\right\}$.

(b) If $\vec{\chi}\left(D_{1}\right)=\vec{\chi}\left(D_{2}\right)=k$ and $k \geqslant 2$, then $\vec{\chi}(D)=k$.

(c) If both $D_{1}$ and $D_{2}$ are $k$-critical and $k \geqslant 2$, then $D$ is $k$-critical.

(d) If $D$ is $k$-critical and $k \geqslant 2$, then both $D_{1}$ and $D_{2}$ are $k$-critical.

Proof. Suppose that $D=\left(D_{1}, v_{1}, u_{1}\right) \nabla\left(D_{2}, v_{2}, u_{2}\right)$ and let $v$ denote the vertex that is obtained from identifying $v_{1}$ and $v_{2}$. To simplify the proof, we assume that $v=v_{1}=v_{2}$. For the proof of (a) let $\vec{\chi}(D)=k$ and let $\varphi$ be a $k$-coloring of $D$. For $i \in\{1,2\}$, let $\varphi_{i}$ denote the restriction of $\varphi$ to $D_{i}$, where $\varphi_{i}\left(v_{i}\right)=\varphi(v)$. We claim that either $\varphi_{1}$ is a $k$-coloring of $D_{1}$ or $\varphi_{2}$ is a $k$-coloring of $D_{2}$. Otherwise, in $D_{1}$ there is a monochromatic directed cycle $C_{1}$ that contains the arc $u_{1} v_{1}$ (as $D_{1}-u_{1} v_{1}$ is a subdigraph of $D$ and therefore $k$-colorable). Similar, in $D_{2}$ there exists a monochromatic cycle $C_{2}$ that contains the arc $v_{2} u_{2}$. But then, $C_{1} \cup C_{2}-u_{1} v_{1}-v_{2} u_{2}+u_{1} u_{2}$ is a monochromatic directed cycle in $D$, a contradiction. This proves (a).

In order to prove $(\mathrm{b})$, let $\vec{\chi}\left(D_{1}\right)=\vec{\chi}\left(D_{2}\right)=k$. By (a), $\vec{\chi}(D) \geqslant k$. Thus, it suffices to show that $\vec{\chi}(D) \leqslant k$. For $i \in\{1,2\}$, let $\varphi_{i}$ be a $k$-coloring of $D_{i}$. By permuting the colors if necessary we obtain $\varphi_{1}\left(v_{1}\right)=\varphi_{2}\left(v_{2}\right)$. For $w \in V(D)$ let

$$
\varphi(w)= \begin{cases}\varphi_{1}(w) \quad \text { if } w \in V\left(D_{1}\right), \\ \varphi_{2}(w) \quad \text { if } w \in V\left(D_{2}\right), \text { and } \\ \varphi_{1}\left(v_{1}\right) \quad \text { if } w=v\end{cases}
$$


We claim that $\varphi$ is a $k$-coloring of $D$. For otherwise, $D$ would contain a monochromatic directed cycle $C$ with $\left\{u_{1}, u_{2}, v\right\} \subseteq V(C)$ and $u_{1} u_{2} \in A(C)$. But then, $\left(C \cap D_{1}\right)+u_{1} v_{1}$ is a monochromatic directed cycle in $D_{1}$, which is impossible.

For the proof of (c) it suffices to show that $\vec{\chi}(D-a)<k$ for all $a \in A(D)$ (by (b)). If $a=u_{1} u_{2}$, then choosing $(k-1)$-colorings of $D_{1}-u_{1} v_{1}$ and $D_{2}-v_{2} u_{2}$ that assign the same color to $v_{1}$ and $v_{2}$ and taking the union of those colorings clearly leads to a $(k-1)$ coloring of $D-a$. Let $a \in A(D) \backslash\left\{u_{1} u_{2}\right\}$. By symmetry, we may assume that $a \in A\left(D_{1}\right)$. Then, there is a $(k-1)$-coloring $\varphi_{1}$ of $D_{1}-a$ and a $(k-1)$-coloring $\varphi_{2}$ of $D_{2}-v_{2} u_{2}$ such that $\varphi_{1}\left(v_{1}\right)=\varphi_{2}\left(v_{2}\right)$. We claim that taking the union of those colorings gives us a $(k-1)$-coloring of $D-a$. Suppose, to the contrary, that there exists a monochromatic cycle $C$ in $D-a$. Then $C$ contains the arc $u_{1} u_{2}$ and $\left(C \cap D_{1}\right)+u_{1} v_{1}$ is a monochromatic cycle in $D_{1}-a$, which is impossible. Hence, $D$ is $k$-critical, as claimed.

To prove statement (d) first assume that $\vec{\chi}\left(D_{1}\right) \leqslant k-1$. Then there is a $(k-1)$ coloring $\varphi_{1}$ of $D_{1}$. Since $D$ is $k$-critical, there furthermore exists a $(k-1)$-coloring $\varphi_{2}$ of $D_{2}-v_{2} u_{2}$ with $\varphi_{2}\left(v_{2}\right)=\varphi_{1}\left(v_{1}\right)$ and the union of $\varphi_{1}$ and $\varphi_{2}$ leads to a $(k-1)$-coloring of $D$ (by the same arguments as in (c)). Hence, $\vec{\chi}\left(D_{1}\right) \geqslant k$ and, by symmetry, we obtain $\vec{\chi}\left(D_{2}\right) \geqslant k$. In order to complete the proof, it suffices to show that $\vec{\chi}\left(D_{i}-a\right)<k$ for $i \in\{1,2\}$ and for $a \in A\left(D_{i}\right)$. By symmetry, we may assume that $i=1$. If $a=u_{1} v_{1}$, then $\vec{\chi}\left(D_{1}-a\right)<k$ as $D_{1}-a$ is a proper subdigraph of $D$ and therefore $(k-1)$-colorable. Let $a \in A\left(D_{1}\right) \backslash\left\{u_{1} v_{1}\right\}$. Then, there is a $(k-1)$-coloring $\varphi$ of $D-a$. We claim that the restriction of $\varphi$ to $V\left(D_{1}\right)$ is a $(k-1)$-coloring of $D_{1}-a$. For otherwise, in $D_{1}-a$ there would exist a monochromatic directed cycle $C_{1}$ that contains the arc $u_{1} v_{1}$. Since $\vec{\chi}\left(D_{2}\right) \geqslant k$, the restriction of $\varphi$ to $V\left(D_{2}\right)$ creates a monochromatic directed cycle $C_{2}$ in $D_{2}$ that contains the arc $u_{2} v_{2}$. However, $C_{1} \cup C_{2}-u_{1} v_{1}-v_{2} u_{2}+u_{1} u_{2}$ is a monochromatic directed cycle in $D-a$ with respect to $\varphi$, a contradiction. This completes the proof.

Another common operation for graphs and digraphs is the identification of independent sets. Let $D$ be a digraph and let $I$ be a non-empty independent set of $D$, i.e., $D[I]$ has no arcs. Then, we can create a new digraph $H$ from $D-I$ by adding a new vertex $v$ and adding all arcs from $v$ to $N_{D}^{+}(I)=\bigcup_{u \in I} N_{D}^{+}(u)$ and all arcs from $N_{D}^{-}(I)=\bigcup_{u \in I} N_{D}^{-}(u)$ to $v$. We say that $H$ is obtained from $D$ by identifying $\boldsymbol{I}$ with $\boldsymbol{v}$, or briefly by identifying independent vertices and write $H=D /(I \rightarrow v)$ (briefly $H=D / I)$. It is obvious that any $k$-coloring of $D / I$ can be extended to a $k$-coloring of $D$ by coloring each vertex of $I$ with the color of $v$. Thus, $\vec{\chi}(D / I) \geqslant \vec{\chi}(D)$.

We define the class of Hajós- $\boldsymbol{k}$-constructible digraphs as the smallest family of digraphs that contains all bidirected complete graphs of order $k$ and is closed under Hajós joins and identifying independent vertices. The class of Hajós- $\boldsymbol{k}$-constructible graphs is defined accordingly. In 1961, Hajós [10] proved the following remarkable result.

Theorem 3 (Hajós). Let $k \geqslant 3$ be an integer. A graph has chromatic number at least $k$ if and only if it contains a Hajós-k-constructible subgraph.

For digraphs, we obtain a similar result. While our proof also uses some of the original ideas from Hajós, we need some new tricks related to perfect digraphs, which are examined below. 
Theorem 4. Let $k \geqslant 3$ be an integer. A digraph has dichromatic number at least $k$ if and only if it contains a Hajós- $k$-constructible subdigraph.

Recall that the clique number $\omega(D)$ of a digraph $D$ is the order of the largest bidirected complete subdigraph of $D$. As $\vec{\chi}\left(D\left(K_{n}\right)\right)=n$, every digraph $D$ satisfies $\omega(D) \leqslant$ $\vec{\chi}(D)$. A perfect digraph is a digraph $D$ satisfying that for each induced subdigraph $D^{\prime}$ of $D$ it holds $\vec{\chi}\left(D^{\prime}\right)=\omega\left(D^{\prime}\right)$. Recall that an odd hole is an (undirected) cycle of odd length at least 5 and an odd antihole is the complement of an odd hole. Moreover, a filled odd hole/filled odd antihole is a digraph $D$ so that $S(D)$ is an odd hole/antihole, where $S(D)$ is the symmetric part of $D$, that is, the graph with vertex set $V(D)$ and edge set

$$
E(S(D))=\{u v \mid u v \in A(D) \text { and } v u \in A(D)\} .
$$

Andres and Hochstättler [1, Corollary 5] proved the following result on perfect digraphs.

Theorem 5 (Andres and Hochstättler). A digraph D is perfect if and only if it contains none of the following as an induced subdigraph: a filled odd hole, a filled odd antihole, and a directed cycle of length at least 3 .

This theorem is a really nice and powerful tool in many ways. For the class of bidirected graphs, the theorem is equivalent to the Strong Perfect Graph Theorem (SPGT) by Chudnovsky, Robertson, Seymour, and Thomas [3], and hence, the SPGT follows from Andres and Hochstättler's result. Nevertheless, their proof heavily relies on the SPGT. We will use their result for the following corollary.

Corollary 6. Let $D$ be a digraph and for $u, v \in V(D)$ let $u \sim v$ denote the relation that $u v \notin A(D)$. If $\sim$ is transitive, then $D$ is perfect.

Proof. By Theorem 5, we only need to prove that $D$ does neither contain a filled odd hole, nor a filled odd antihole, nor an induced directed cycle of length at least 3 as an induced subdigraph.

First assume that $D$ contains a filled odd hole $C$ as an induced subdigraph. Let $v_{1}, v_{2}, \ldots, v_{r}, v_{1}$ be a cyclic ordering of the vertices of the filled odd hole. Then $r$ is odd and $r \geqslant 5$. By symmetry, we may assume that $v_{3} \sim v_{1}$. As $\sim$ is transitive, this implies that $v_{1} v_{4} \in A(D)$ (as otherwise $v_{3} \sim v_{1}, v_{1} \sim v_{4}$, but $v_{3} \nsim v_{4}$ ) and so $v_{4} \sim v_{1}$. As a consequence, $v_{1} v_{3} \in A(D)$ (since $v_{4} \sim v_{1}$ and $v_{4} v_{3} \in A(D)$ ). By continuing this argumentation we obtain that $v_{1} v_{i} \in A(D)$ for all $i \in\{2,3, \ldots, r\}$. Moreover, regarding $v_{2}$, it follows that $v_{2} v_{4} \in A(D)$ (as otherwise $v_{2} \sim v_{4}, v_{4} \sim v_{1}$, but $v_{2} \not v_{1}$, a contradiction). As a consequence, $v_{2} v_{i} \in A(D)$ for all $i \in\{4,5, \ldots, r\}$. Finally, $v_{3} v_{r} \in A(D)$ (as otherwise $v_{3} \sim v_{r}, v_{r} \sim v_{2}$, but $v_{3} \not v_{2}$ ). However, since $C$ is a filled odd hole, this gives us $v_{r} \sim v_{3}$ and so $v_{r} \sim v_{3}, v_{3} \sim v_{1}$, but $v_{1} \nsim v_{r}$, a contradiction. Thus, $D$ cannot contain a filled odd hole as an induced subdigraph.

Next assume that $D$ contains a filled odd antihole $C$ as an induced subdigraph. Let again $v_{1}, v_{2}, \ldots, v_{r}, v_{1}$ be a cyclic ordering of the vertices. Then $r$ is odd and $r \geqslant 5$. By symmetry, we may assume that $v_{1} \sim v_{2}$. Then, $v_{2} v_{3} \in A(D)$ as otherwise $\sim$ would not be transitive. Continuing this argument, we obtain that $v_{i} \sim v_{i+1}$ for $i$ odd and $v_{i} v_{i+1} \in A(D)$ 
for even $i$. As $r$ is odd this implies $v_{r} \sim v_{1}$. As a consequence, $v_{r} \sim v_{1}, v_{1} \sim v_{2}$, but $v_{r} v_{2} \in A(D)$, a contradiction. Thus, $D$ contains no filled antiholes as induced subdigraphs.

Finally, assume that $D$ contains an directed cycle $C$ of length at least 3 as an induced subdigraph. Again, let $v_{1}, v_{2}, \ldots, v_{r}, v_{1}$ be a cyclic ordering of the vertices of $C$. Then, $v_{1} \sim v_{r}, v_{r} \sim v_{2}$, but $v_{1} v_{2} \in A(D)$, a contradiction. As a consequence, $D$ is perfect by Theorem 5 , and we are done.

Proof of Theorem 4. Let $k \geqslant 3$ be an integer. Clearly, every Hajós- $k$-constructible digraph has dichromatic number at least $k$ (by Theorem 2 and since $\vec{\chi}(D / I) \geqslant \vec{\chi}(D)$ for each independent set $I$ of a digraph $D$ ). This proves the "if"-implication. The proof of the "only if"-implication is by reductio ad absurdum. Let $D$ be a maximal counter-example in the sense that $\vec{\chi}(D) \geqslant k$ and $D$ does not contain a Hajós- $k$-constructible subdigraph, but adding a new arc $a \in A(\bar{D})$ to $D$ implies the existence of a Hajós- $k$-constructible subdigraph $D_{a}$ of $D+a$ with $a \in A\left(D_{a}\right)$. For two vertices $u, v \in V(D)$, let $u \sim v$ denote the relation that $u v \notin A(D)$. We distinguish between two cases and show that both of them lead to a contradiction.

Case 1: $\sim$ is transitive. Then, $D$ is perfect by Corollary 6 and so $D$ contains a bidirected complete graph of order at least $k$ as a subdigraph and, therefore, a Hajós- $k$-constructible sudigraph, which is impossible.

Case 2: $\sim$ is not transitive. Then there are vertices $u, v, w \in V(D)$ such that $u v \notin$ $A(D), v w \notin A(D)$, but $u w \in A(D)$. Hence, both arcs $u v$ and $v w$ belong to $A(\bar{D})$. By the maximality of $D$, there exist Hajós- $k$-constructible subdigraphs $D_{u v} \subseteq D+u v$ and $D_{v w} \subseteq D+v w$ with $u v \in A\left(D_{u v}\right)$ and $v w \in A\left(D_{v w}\right)$. Let $D^{\prime}$ be the graph obtained from the union $\left(D_{u v}-u v\right) \cup\left(D_{v w}-v w\right)$ by adding the arc $u w$. Then, $D^{\prime}$ is a subdigraph of $D$ that can be obtained from disjoint copies of $D_{u v}$ and $D_{v w}$ as follows. First we apply the Hajós join by removing the copies of the arcs $u v$ and $v w$, identifying the two copies of $v$, and adding the arc from $u \in V\left(D_{u v}\right)$ to $w \in V\left(D_{v w}\right)$. Afterwards, for each vertex $x$ that belongs to both $D_{u v}$ and $D_{v w}$, we identify the two copies of $x$. Hence, $D^{\prime}$ is a Hajós- $k$-constructible subdigraph of $D$, a contradiction. This completes the proof.

While reading our submission, one of the referees came up with another, very nice idea for proving Corollary 6 using Dilworth's Theorem. Recall that a preorder $P=(X, \prec)$ consists of a set $X$ and a binary relation $\prec$, which is reflexive and transitive. Two elements $x, y \in X$ are comparable (with respect to $P$ ) if $x \prec y$ or $y \prec x$ and incomparable, otherwise. A chain in $P$ is a subset $Y \subseteq X$ of pairwise comparable elements, an antichain on $P$ is a subset $Z \subseteq X$ of pairwise incomparable elements. The well known theorem of Dilworth [4] (see also [2, Theorem 13.5.8]) states the following.

Theorem 7 (Dilworth). Let $P=(X, \prec)$ be a preorder. Then the minimum number of chains needed to cover $X$ equals the maximum number of elements in an antichain.

Alternate Proof of Corollary 6. Let $D^{\prime} \subseteq D$ be an induced subdigraph of $D$ and let $\vec{\chi}\left(D^{\prime}\right)=k$. We claim that $\omega\left(D^{\prime}\right)=k$. Recall that for vertices $u, v \in V(D), u \sim v$ denotes the relation that $u v \notin A(D)$. Since $\sim$ is transitive on $A(D)$, the relation $\sim$ is transitive on $A\left(D^{\prime}\right)$, and so $P=\left(V\left(D^{\prime}\right), \sim\right)$ is a preorder. Then, an antichain on $P$ 
induces a bidirected complete graph in $D^{\prime}$ (as $u, v \in V\left(D^{\prime}\right)$ are incomparable if and only if $u v \in A\left(D^{\prime}\right)$ and $\left.v u \in A\left(D^{\prime}\right)\right)$. Furthermore, it is easy to see that $Y$ is a chain in $P$ if and only if $D^{\prime}[Y]$ is an acyclic subdigraph of $D^{\prime}$. Hence, a cover of $V\left(D^{\prime}\right)$ with $\ell$ chains corresponds to an $\ell$-coloring of $D^{\prime}$. As $\chi\left(D^{\prime}\right)=k$, we need $k$ chains in order to cover $V\left(D^{\prime}\right)$ and so there is an antichain $Z \subseteq V\left(D^{\prime}\right)$ of order $k$, i.e. $D^{\prime}[Z]=D\left(K_{k}\right)$. Thus, $\omega\left(D^{\prime}\right) \geqslant k$ and, as $\vec{\chi}\left(D^{\prime}\right)=k$, we have $\omega\left(D^{\prime}\right)=k$. Consequently, $D$ is perfect.

A third short proof of Corollary 6 can be obtained by applying the Gallai-Milgram Theorem [6] (see also [2, Theorem 13.5.2]) to the complement $\bar{D}$ of $D$.

In the last two decades Hajós' theorem (Theorem 3) became very popular among graph theorists. Hajós-like theorems were established for the list chromatic number by Gravier [9] and Král [20], for the circular chromatic number by Zhu [34], for the signed chromatic number by Kang [18], for the chromatic number of edge weighted graphs by Mohar [23], for graph homomorphisms by Nešetril [26], and for Grassmann homomorphisms (a homomorphism concept that provides a common generalization of graph colorings, hypergraph colorings and nowhere-zero flows) by Jensen [16].

\section{The Ore construction}

Regarding undirected graphs, Urquhart [33] proved that each graph with chromatic number at least $k$ does not only contain a Hajós- $k$-constructible subgraph but itself is Hajós$k$-constructible. The aim of this section is to point out that the same result does not hold for digraphs and to prove that, however, a slight modification of the Hajós join does the trick.

Theorem 8. Let $k \geqslant 3$ be an integer and let D be a Hajós-k-constructible digraph. Then, $D$ is strongly connected.

Proof. Clearly, if $D$ is a strongly connected digraph, then identifying non-adjacent vertices still leads to a strongly connected digraph. Moreover, if $D_{1}$ and $D_{2}$ are strongly connected, then the directed Hajós-join of $D_{1}$ and $D_{2}$ is strongly connected, too, as vertices on directed cycles are still on directed cycles after the Hajós join (consider Figure 1 for a visualization).

As a consequence of the above theorem, every digraph with dichromatic number at least $k$ that is not strongly connected is not Hajós- $k$-constructible and so Urquhart's Theorem cannot be directly transferred to digraphs. Nevertheless, it turns out that we get an Urquhart-type theorem by further allowing the following join. Let $D_{1}$ and $D_{2}$ be two digraphs and let $u_{1}, v_{1} \in V\left(D_{1}\right)$ and $u_{2}, v_{2} \in V\left(D_{2}\right)$ such that $D_{i}\left[\left\{u_{i}, v_{i}\right\}\right]$ is a digon for $i \in\{1,2\}$. Now let $D$ be the digraph obtained from the union $D_{1} \cup D_{2}$ by deleting both arcs between $u_{1}$ and $v_{1}$ as well as both arcs between $u_{2}$ and $v_{2}$, identifying the vertices $v_{1}$ and $v_{2}$ to a new vertex $v$, and adding both $\operatorname{arcs} u_{1} u_{2}$ and $u_{2} u_{1}$. We say that $D$ is the bidirected Hajós join of $D_{1}$ and $D_{2}$ and write $D=\left(D_{1}, v_{1}, u_{1}\right) \stackrel{\leftrightarrow}{\nabla}\left(D_{2}, v_{2}, u_{2}\right)$ or, briefly, $D=D_{1} \stackrel{\leftrightarrow}{\nabla} D_{2}$. Note that the bidirected Hajós join is the exact analogue of the undirected 
Hajos join. By a slight modification of the proof of Theorem 2(a)-(c) one can easily show that the following holds.

Theorem 9 (Bidirected Hajós Construction). Let $D=D_{1} \stackrel{\leftrightarrow}{\nabla} D_{2}$ result from the bidirected Hajós join of two disjoint non-empty digraphs $D_{1}$ and $D_{2}$. Then, the following statements hold:

(a) $\vec{\chi}(D) \geqslant \min \left\{\vec{\chi}\left(D_{1}\right), \vec{\chi}\left(D_{2}\right)\right\}$.

(b) If $\vec{\chi}\left(D_{1}\right)=\vec{\chi}\left(D_{2}\right)=k$ and $k \geqslant 3$, then $\vec{\chi}(D)=k$.

(c) If both $D_{1}$ and $D_{2}$ are $k$-critical and $k \geqslant 3$, then $D$ is $k$-critical.

Note that for the proof of statement (b), we use the fact that $k \geqslant 3$ and so we can choose $\varphi_{1}$ and $\varphi_{2}$ such that $\varphi_{1}\left(v_{1}\right)=\varphi_{2}\left(v_{2}\right)$ and $\varphi_{1}\left(u_{1}\right) \neq \varphi_{2}\left(u_{2}\right)$. For $k=2$, the statement is not true: for example, $D\left(C_{4}\right) \stackrel{\leftrightarrow}{\nabla} D\left(C_{4}\right)=D\left(C_{7}\right)$, whereas $\vec{\chi}\left(D\left(C_{4}\right)\right)=2 \neq$ $3=\vec{\chi}\left(D\left(C_{7}\right)\right)$. The same trick works for statement (c).

For the proof of his Theorem, Urquhart even used a more restricted class of constructible (undirected) graphs than the class of Hajós- $k$-constructible graphs, which originally was introduced by Ore [29, Chapter 11]. Transferred to digraphs, we get the following. Let $D_{1}$ and $D_{2}$ be two vertex-disjoint digraphs, let $u_{1} v_{1}$ be an arc of $D_{1}$, and let $v_{2} u_{2}$ be an arc of $D_{2}$. Furthermore, let $\iota: S_{1} \rightarrow S_{2}$ be a bijection with $S_{i} \subseteq V\left(G_{i}-v_{i}\right)$ for $i \in\{1,2\}$ and $\iota\left(u_{1}\right) \neq u_{2}$. Let $D$ be the digraph obtained from $\left(D_{1}, v_{1}, u_{1}\right) \nabla\left(D_{2}, v_{2}, u_{2}\right)$ by identifying $w$ with $\iota(w)$ for each $w \in S_{1}$. Then, $D$ is a directed Ore join of $D_{1}$ and $D_{2}$ and we write $D=\left(D_{1}, v_{1}, u_{1}\right) \nabla_{\iota}^{o}\left(D_{2}, v_{2}, u_{2}\right)$. Note that the undirected Ore join of two undirected graphs $G_{1}$ and $G_{2}$ is performed via an undirected Hajós join and identification afterwards. However, for digraphs we need a second type of Ore join: If $u_{1}, v_{1} \in V\left(D_{1}\right)$ and $u_{2}, v_{2} \in V\left(D_{2}\right)$ are vertices such that $D_{i}\left[\left\{u_{i}, v_{i}\right\}\right]$ is a digon for $i \in\{1,2\}$ and if $\iota$ is the bijection from above, then the digraph $D$ obtained from $\left(D_{1}, v_{1}, u_{1}\right) \stackrel{\leftrightarrow}{\nabla}\left(D_{2}, v_{2}, u_{2}\right)$ by identifying $w$ with $\iota(w)$ for each $w \in S_{1}$ is a bidirected Ore join of $D_{1}$ and $D_{2}$ and we write $D=\left(D_{1}, v_{1}, u_{1}\right) \stackrel{\leftrightarrow}{\nabla}_{\iota}^{o}\left(D_{2}, v_{2}, u_{2}\right)$. Recall that if the identification would lead to more than one arc in the same direction between two vertices, all but one of those arcs get deleted.

We define the class of Ore- $\boldsymbol{k}$-constructible digraphs as the smallest family of digraphs that contains all bidirected complete graphs of order $k$ and is closed under both directed and bidirected Ore joins. The proof of Theorem 4 immediately implies the following theorem (see [29] for the undirected analogue). In particular, here we do not need any bidirected Ore joins.

Theorem 10. Let $k \geqslant 3$ be an integer. A digraph has dichromatic number at least $k$ if and only if it contains an Ore-k-constructible subdigraph.

Urquhart [33] proved the following result, thereby answering a conjecture by Hanson, Robinson, and Toft [11] (the conjecture was also proposed by Jensen and Toft in their book on graph coloring problems [17, Problem 11.5]). 
Theorem 11 (Urquhart). Let $k \geqslant 3$ be an integer. For a graph $G$ the following conditions are equivalent:

(a) $G$ satisfies $\chi(G) \geqslant k$.

(b) $G$ is Hajós-k-constructible.

(c) $G$ is Ore-k-constructible.

Note that if $G$ is the Hajós join of two graphs $G_{1}$ and $G_{2}$, then $D(G)$ is the bidirected Hajós join of $D\left(G_{1}\right)$ and $D\left(G_{2}\right)$. Furthermore, $\vec{\chi}(D(G))=\chi(G)$ and so the above theorem immediately implies the following.

Observation 12. Each bidirected graph with dichromatic number at least $k \geqslant 3$ is Ore$k$-constructible.

Now we have all the tools that we need in order to prove our Urquhart-type theorem.

Theorem 13. Let $k \geqslant 3$ be an integer. A digraph has dichromatic number at least $k$ if and only if it is Ore-k-constructible.

Proof. It immediately follows from Theorem 2(a) and Theorem 9 (a) that each Ore- $k$ constructible digraph has dichromatic number at least $k$.

Thus, it suffices to show that each digraph with dichromatic number at least $k$ is Ore$k$-constructible. We will do this via a sequence of claims. In the following, we will denote by $D\left(K_{k}\right)+\vec{v}$ (respectively $D\left(K_{k}\right)+\overleftarrow{v}$ ) the digraph that results from $D\left(K_{k}\right)$ by adding a new vertex $v$ and the arc $u v$ (respectively $v u$ ) for some vertex $u$ of $D\left(K_{k}\right)$. Moreover, let $D\left(K_{k}\right)+a$ be the digraph that results from $D\left(K_{k}\right)$ by adding two new vertices $u, v$ and the arc $a=u v$. Finally, $\mathcal{O}_{k}$ denotes the class of Ore- $k$-constructible digraphs and $\mathcal{O}_{k}^{*}$ denotes the class of Ore- $k$-constructible digraphs containing a bidirected complete graph of order $k$. It follows from Observation 12 that

Claim 1. The digraph obtained from $D\left(K_{k}\right)$ by adding an isolated vertex belongs to $\mathcal{O}_{k}^{*}$.

Claim 2. The digraph $D\left(K_{k}\right)+$ a belongs to $\mathcal{O}_{k}^{*}$.

Proof. It is clear that $D\left(K_{k}\right)+a$ still contains a copy of $D\left(K_{k}\right)$. We claim that $D\left(K_{k}\right)+a$ is Ore-constructible. To this end, let $D_{1}$ (respectively $D_{2}$ ) be the bidirected graph obtained by identifying a vertex of $D\left(K_{k}\right)$ to a vertex of a disjoint copy of $D\left(K_{2}\right)$ (respectively $\left.D\left(K_{3}\right)\right)$. More formally,

$$
\begin{aligned}
V\left(D_{1}\right) & =\left\{v_{1}, v_{2}, \ldots, v_{k}, u\right\}, \\
A\left(D_{1}\right) & =\left\{v_{i} v_{j} \mid i \neq j\right\} \cup\left\{v_{1} u, u v_{1}\right\}, \\
V\left(D_{2}\right) & =\left\{v_{1}^{\prime}, v_{2}^{\prime}, \ldots, v_{k}^{\prime}, u_{1}, u_{2}\right\}, \text { and } \\
A\left(D_{2}\right) & =\left\{v_{i}^{\prime} v_{j}^{\prime} \mid i \neq j\right\} \cup\left\{v_{1}^{\prime} u_{1}, v_{1}^{\prime} u_{2}, u_{1} v_{1}^{\prime}, u_{2} v_{1}^{\prime}, u_{1} u_{2}, u_{2} u_{1}\right\}
\end{aligned}
$$

(see Figure 2a). Let $\iota$ be the bijection with $\iota\left(v_{i}\right)=v_{i}^{\prime}$ for all $i \in\{1,2, \ldots, k\}$ and let $D_{2}^{\prime}=\left(D_{1}, u, v_{1}\right) \nabla_{\iota}^{o}\left(D_{2}, u_{2}, u_{1}\right)$ (see Figure 2(a) and (b)). This Ore-join leads to the 

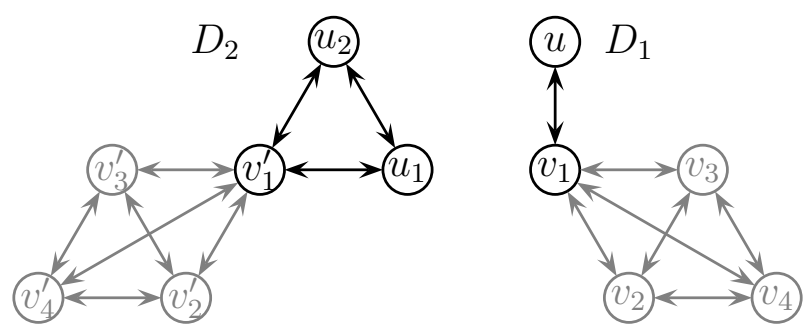

(a) The digraphs $D_{2}$ and $D_{1}$. Firstly, we perform the directed Hajós join $\left(D_{1}, u, v_{1}\right) \Delta\left(D_{2}, u_{2}, u_{1}\right)$.

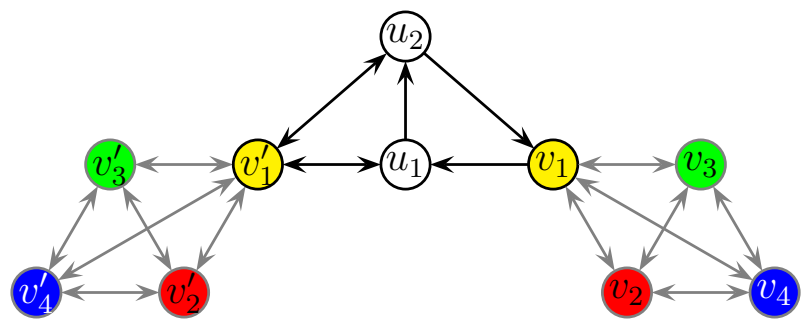

(b) The graph we obtain after the Hajós join. Now, we identify the vertices of the same color.

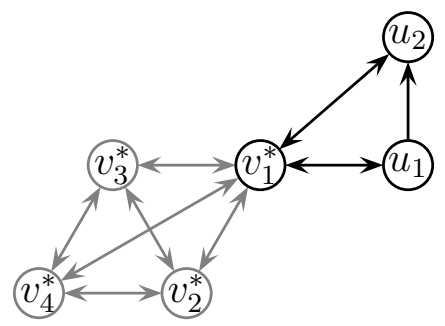

(c) This gives us the graph $D_{2}^{\prime}$.

Figure 2: The first step of the construction of Claim 2.

digraph $D_{2}^{\prime}=D_{2}-u_{2} u_{1}$ (see Figure 2(c)). By $v_{i}^{*}$ we denote the vertex that results from identifying $v_{i}$ with $\iota\left(v_{i}\right)=v_{i}^{\prime}$.

Now we take a new copy of $D_{1}$, define $\iota^{\prime}$ to be the bijection with $\iota^{\prime}\left(v_{i}^{*}\right)=v_{i+1}$ for all $i \in\{1,2, \ldots, k\}$ (where $v_{k+1}=v_{1}$ ), and set $D_{2}^{\prime \prime}=\left(D_{2}^{\prime}, u_{1}, v_{1}^{*}\right) \stackrel{\leftrightarrow}{\nabla}_{\iota^{\prime}}^{o}\left(D_{1}, u, v_{1}\right)$ (see Figure $3 \mathrm{~b}$ ). Still, let $v_{i}^{*}$ denote the vertex that results from identifying $v_{i}^{*}$ with $\iota^{\prime}\left(v_{i}^{*}\right)$.

Finally, we take another copy of $D_{1}$, set $\iota^{\prime \prime}\left(v_{i}^{*}\right)=v_{i+1}$ for $i \in\{1,2, \ldots, k\}$ (where $\left.v_{k+1}=v_{1}\right)$ and perform the Ore join $\left(D_{2}^{\prime \prime}, u_{2}, v_{1}^{*}\right) \stackrel{\leftrightarrow}{\nabla}_{\iota^{\prime \prime}}^{o}\left(D_{1}, u, v_{1}\right)$ (see Figure $\left.4(\mathrm{a})(\mathrm{b})\right)$. This gives us the digraph $D\left(K_{k}\right)+u_{1} u_{2}$ as required. 


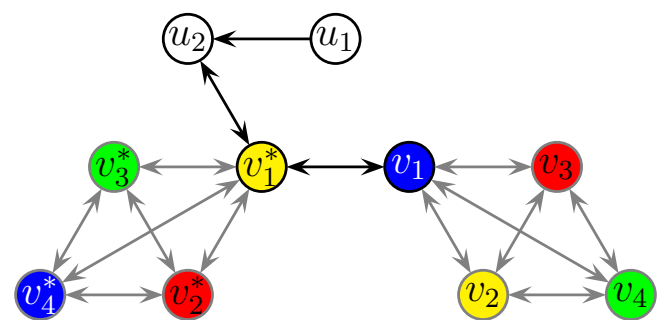

(a)

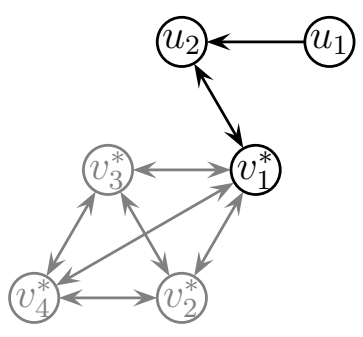

(b)

Figure 3: How to build the graph $D_{2}^{\prime \prime}$.

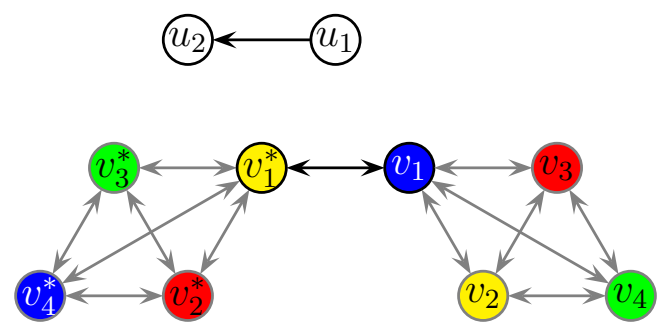

(a)
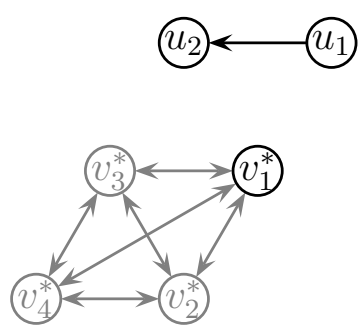

(b)

Figure 4: The final step of the construction.

Claim 3. The digraphs $D\left(K_{k}\right)+\vec{v}$ and $D\left(K_{k}\right)+\overleftarrow{v}$ are in $\mathcal{O}_{k}^{*}$

Proof. For the exact construction of $D\left(K_{k}\right)+\vec{v}$ see Figure 5; we start with the graphs that results from $D\left(K_{k}\right)$ by adding a vertex $v$ and joining it to either one or two vertices of $D\left(K_{k}\right)$ by arcs in both directions. The construction of $D\left(K_{k}\right)+\overleftarrow{v}$ can be obtained (by symmetry) by changing the order of the digraphs in the directed Hajós join in Figure 5(a).

From now on, we may argue similar to the original proof of Urquhart.

Claim 4. Let $D$ be a digraph belonging to $\mathcal{O}_{k}^{*}$. Then, the digraph $D^{\prime}$ obtained from $D$ by adding an isolated vertex belongs to $\mathcal{O}_{k}^{*}$, too.

Proof. It suffices to show that $D^{\prime} \in \mathcal{O}_{k}$. Let $D_{2}$ be a copy of $D\left(K_{k}\right)$ plus an isolated vertex (which belongs to $\mathcal{O}_{k}^{*}$ by Claim 1 ). As $D^{\prime} \in \mathcal{O}_{k}^{*}$, there is a vertex set $X_{1} \subseteq V(D)$ such that $D\left[X_{1}\right]$ is isomorphic to $D\left(K_{k}\right)$. Let $X_{2}$ be the vertex set of the bidirected complete graph of order $k$ contained in $D_{2}$ and, for $i \in\{1,2,3\}$, let $v_{i}, w_{i}, u_{i}$ be three vertices of $X_{i}$. Furthermore, let $\iota: X_{1} \backslash\left\{v_{1}\right\} \rightarrow X_{2} \backslash\left\{v_{2}\right\}$ be a bijection such that $\iota\left(u_{1}\right)=w_{2}$ and $\iota\left(w_{1}\right)=u_{2}$. Then, $D^{\prime}=\left(D, v_{1}, u_{1}\right) \stackrel{\leftrightarrow}{\nabla}_{\iota}^{o}\left(D_{2}, v_{2}, u_{2}\right)$ and we are done. 

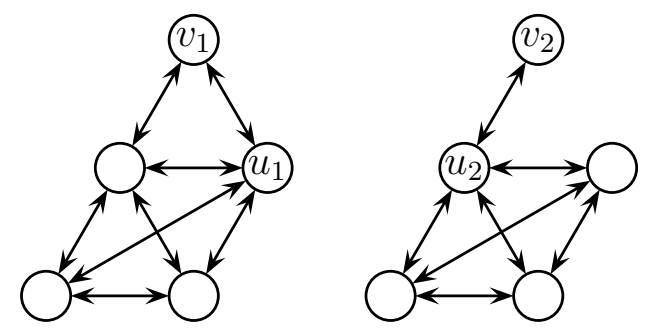

(a) We start by performing a directed Hajós join between the two depicted graphs. These are in $\mathcal{O}_{k}^{*}$ by Theorem 11 .

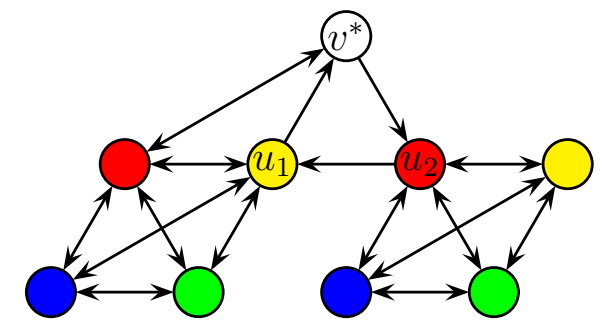

(b) Afterwards, we identify the vertices of the same color.

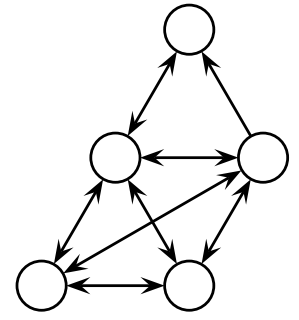

(c) End of the first step.
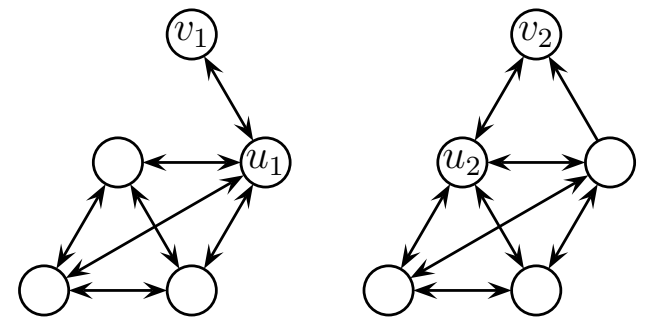

(d) Now we perform a bidirected Hajós join.

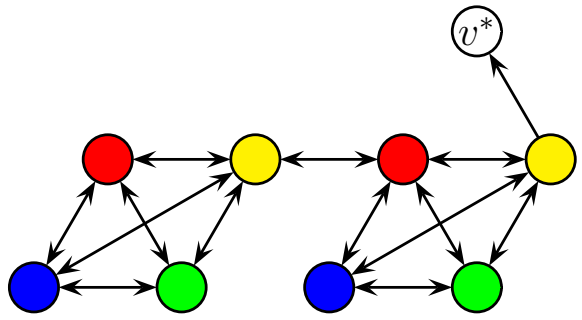

(e) Again we identify vertices of the same color.

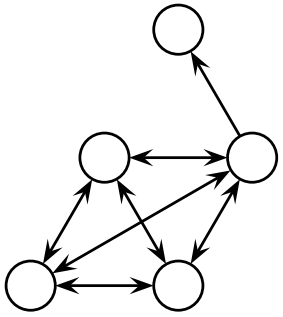

(f) We get the digraph $D\left(K_{k}\right)+\vec{v}$.

Figure 5: The construction of Claim 3.

Claim 5. Let $D$ be a digraph belonging to $\mathcal{O}_{k}^{*}$ and let $a \in A(\bar{D})$. Then, the digraph $D+a$ belongs to $\mathcal{O}_{k}^{*}$, too.

Proof. Since $D \in \mathcal{O}_{k}^{*}$, there is a vertex set $X \subseteq V(D)$ such that $D[X]$ is a copy of $D\left(K_{k}\right)$. We distuingish between two cases. 
Case 1: One end-vertex of the arc a belongs to $X$. Then, we may assume $a=u v$ with $u \in X$ and $v \in V \backslash X$ (the case $a=v u$ can be done analogously). Moreover, let $D^{\prime}$ be a copy of $D\left(K_{k}\right)+\overrightarrow{v^{\prime}}$, let $X^{\prime}=V\left(D^{\prime}\right) \backslash\left\{v^{\prime}\right\}$, and let $u^{\prime}$ be the vertex adjacent to $v^{\prime}$ in $D^{\prime}$. Finally, let $w, z \in X \backslash\{u\}$ and let $w^{\prime}, z^{\prime} \in X^{\prime} \backslash\left\{u^{\prime}\right\}$. By Claim $3, D^{\prime} \in \mathcal{O}_{k}$. Now let $\iota$ be a bijection from $(X \backslash\{u\}) \cup\{v\}$ to $\left(X^{\prime} \backslash\left\{u^{\prime}\right\}\right) \cup\left\{v^{\prime}\right\}$ with $\iota(v)=v^{\prime}, \iota(w)=z^{\prime}$, and $\iota(z)=w^{\prime}$. Then, $(D, u, w) \stackrel{\leftrightarrow}{\nabla_{\iota}^{o}}\left(D^{\prime}, u^{\prime}, w^{\prime}\right) \in \mathcal{O}_{k}$ is a copy of $D+a$, and we are done.

Case 2: No end-vertex of a belongs to $X$. Then, let $a=u v$, and $D^{\prime}$ be a copy of $D\left(K_{k}\right)+u^{\prime} v^{\prime}$. By Claim 2, $D^{\prime}$ belongs to $\mathcal{O}_{k}$. Now let $x, y, z$ be three vertices from $X$ and let $\left\{x^{\prime}, y^{\prime}, z^{\prime}\right\} \subseteq D^{\prime} \backslash\{u, v\}$. Finally, let $\iota$ be a bijection from $X \backslash\{x\} \cup\{u, v\}$ to $D^{\prime} \backslash\left\{x^{\prime}\right\}$ with $\iota(u)=u^{\prime}, \iota(v)=v^{\prime}, \iota(y)=z^{\prime}$, and $\iota(z)=y^{\prime}$. Then, $(D, x, y) \stackrel{\leftrightarrow}{\nabla}_{\iota}^{o}\left(D^{\prime}, x^{\prime}, y^{\prime}\right) \in \mathcal{O}_{k}$ is a copy of $D+a$ and the proof of the claim is complete.

It follows from Claims 4 and 5 that each digraph containing $D\left(K_{k}\right)$ belongs to $\mathcal{O}_{k}^{*}$. The remaining part of the proof is by reductio ad absurdum. Let $D$ be a maximal counterexample on a fixed number of vertices in the sense that $\vec{\chi}(D) \geqslant k, D$ is not Ore- $k$ constructible, and $D$ has maximum number of edges with respect to this property. Then, $D$ does not contain $D\left(K_{k}\right)$ and if $a \in A(\bar{D}), D+a$ belongs to $\mathcal{O}_{k}$. Now we argue as in the proof of Theorem 4. For two vertices $u, v \in V(D)$, let $u \sim v$ denote the relation that $u v \notin A(D)$. If $\sim$ is transitive we again conclude from Corollary 6 that $D$ is perfect and, hence, contains $D\left(K_{k}\right)$, a contradiction. Hence, $\sim$ is not transitive and so there are vertices $u, v, w \in V(D)$ with $u v \notin A(D), v w \notin A(D)$, but $u w \in A(D)$. Then, both digraphs $D+u v$ as well as $D+v w$ belong to $\mathcal{O}_{k}$ and $D$ is the Ore join of two disjoint copies of these two digraphs. Thus, $D$ belongs to $\mathcal{O}_{k}$, a contradiction.

\section{A Gallai-type theorem for critical digraphs}

Let $D$ be a $k$-critical digraph. If $v \in V(D)$, then $D-v$ admits a $(k-1)$-coloring and, since $\vec{\chi}(D)=k, v$ must have an out- and an in-neighbor in each color class of such a coloring. Hence, we have $k-1 \leqslant \min \left\{d_{D}^{+}(v), d_{D}^{-}(v)\right\}$ for every vertex $v \in V(D)$, which gives us a natural way to classify the vertices of $D$. We say that a vertex $v \in V(D)$ is a low-vertex of $D$ if $d_{D}^{+}(v)=d_{D}^{-}(v)=k-1$ and a high vertex of $D$, otherwise. Furthermore, let $D_{L}$ denote the digraph that is induced by the set of low vertices of $D$; we will call it the low vertex subdigraph of $D$. For undirected graphs, Gallai [7] proved that the blocks of the low vertex subgraph have a specific structure:

Theorem 14 (Gallai). Let $G_{L}$ be the low vertex subgraph of a $k$-critical graph $G$, i.e., $G_{L}$ is the subgraph of $G$ induced by the vertices having degree $k-1$ in $G$. Then, each block $B$ of $G_{L}$ is a complete graph or an odd cycle.

In the digraph setting, we obtain the following theorem.

Theorem 15. Let $D_{L}$ be the low vertex subdigraph of a $k$-critical digraph $D$. Then, each block $B$ of $D_{L}$ satisfies at least one of the following statements: 
(a) B consists of just one single arc.

(b) $B$ is a directed cycle of length $\geqslant 2$.

(c) $B$ is a bidirected cycle of odd length.

(d) $B$ is a bidirected complete graph.

For the proof of Theorem 15 we will use a theorem of Harutyunyan and Mohar [14] concerning list-colorings of digraphs. Given a digraph $D$, a list-assignment $L$ is a function that assigns each vertex $v \in V(D)$ a set (list) $L(v)$ of colors. An $L$-coloring of $D$ is a coloring $\varphi$ of $D$ such that $\varphi(v) \in L(v)$ for all $v \in V(D)$.

Theorem 16 (Harutyunyan and Mohar). Let $D$ be a connected digraph, and let $L$ be a list-assignment such that $|L(v)| \geqslant \max \left\{d_{D}^{+}(v), d_{D}^{-}(v)\right\}$ for all $v \in V(D)$. Suppose that $D$ has no L-coloring. Then, $D$ is Eulerian and for every block $B$ of $D$ at least one of the following cases occurs:

(a) $B$ is a directed cycle of length $\geqslant 2$.

(b) $B$ is a bidirected cycle of odd length $\geqslant 3$.

(c) B is a bidirected complete graph.

The next proposition states some important facts that will be needed for the proof of Theorem 15.

Proposition 17. Let $D_{L}$ be the low vertex subdigraph of a $k$-critical digraph $D$. Moreover, given a vertex $v \in V\left(D_{L}\right)$, let $\varphi$ be a $(k-1)$-coloring of $D-v$ with color set $\Gamma=$ $\{1,2, \ldots, k-1\}$. Then the following statements hold:

(a) Each color from $\Gamma$ appears exactly once in $N_{D}^{+}(v)$ and in $N_{D}^{-}(v)$.

(b) If $u \in V\left(D_{L}\right)$ is adjacent to $v$, then uncoloring $u$ and coloring $v$ with the color of $u$ leads to a $(k-1)$-coloring of $D-u$.

Proof. Suppose (by symmetry) that there is a color $\alpha \in \Gamma$ such that $\alpha$ does not appear in $N_{D}^{+}(v)$. Then, coloring $v$ with $\alpha$ cannot create a monochromatic cycle in $D$ (as $v$ has no out-neighbor with color $\alpha$ ) and, thus, $D$ would be $(k-1)$-colorable, a contradiction. As $d_{D}^{+}(v)=k-1=|\Gamma|$, this proves (a).

For the proof of (b), assume (by symmetry) that $u v \in A(D)$. Then it follows from (a) that after uncoloring $u, v$ has no in-neighbor with color $\varphi(u)$ and so coloring $v$ with color $\varphi(u)$ cannot create a monochromatic cycle.

In the following, we will call the procedure that is described in Proposition 17(b) shifting the color from $u$ to $v$ and briefly write $u \rightarrow v$. Now let $D$ be a $k$-critical digraph, let $C$ be a (not necessarily directed) cycle in $D_{L}$ and let $v \in V(C)$. Moreover, let $\varphi$ be a $(k-1)$-coloring of $D-v$ and let $u$ and $w$ be the vertices such that $u, v$ and $w$ are 
consecutive in $C$. Then, beginning with $u \rightarrow v$, we can shift each vertex of $C$, one after another, clockwise and obtain a new $(k-1)$-coloring of $D-v$ (see Figure 6 ). Similar, beginning with $w \rightarrow v$, we can shift each vertex of $C$ counter-clockwise and obtain a third $(k-1)$-coloring of $D-v$. The main idea for this goes back to Gallai [7]; we will use this observation frequently in the following.
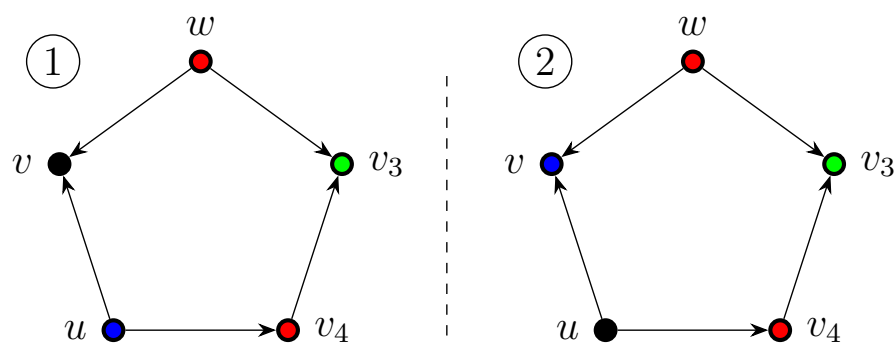

$$
u \rightarrow v
$$

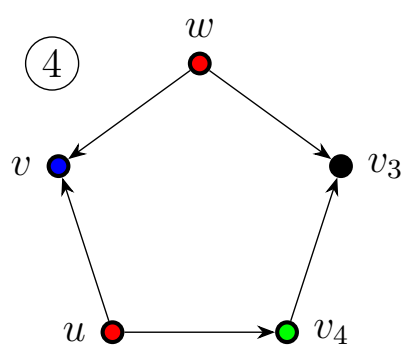

$v_{3} \rightarrow v_{4}$

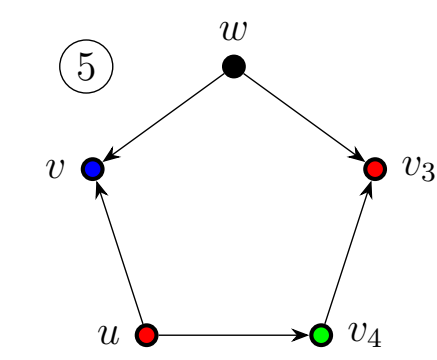

$w \rightarrow v_{3}$

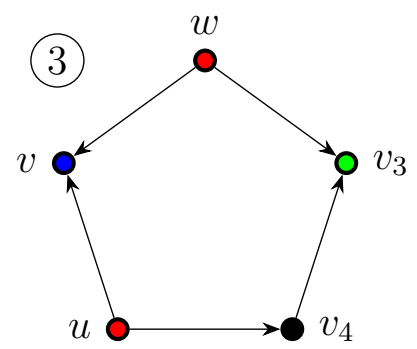

$v_{4} \rightarrow u$

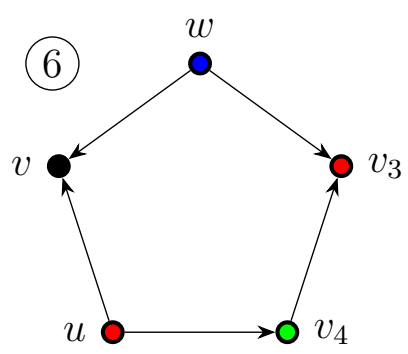

$v \rightarrow w$

Figure 6: The black (uncolored) vertex denotes the clockwise shifting around a cycle.

Proof of Theorem 15. Let $D_{L}$ be the low vertex subdigraph of a $k$-critical digraph $D$ and let $B$ be an arbitrary block of $D_{L}$. If $|B|=1$, then $B=D\left(K_{1}\right)$ and we are done. If $|B|=2$, then either $B$ consists of just one arc or $B$ is a bidirected complete graph and so there is nothing to show. Thus, we may assume $|B| \geqslant 3$.

Claim 1. For all vertices $v \in V(B)$ we have $d_{B}^{+}(v)=d_{B}^{-}(v)$, i.e., $B$ is Eulerian.

Proof. For otherwise, we may assume that $d_{B}^{+}(v)<d_{B}^{-}(v)$ for some $v \in V(B)$. Let $\varphi$ be a $(k-1)$-coloring of $D-v$. Since $d_{D}^{+}(v)=d_{D}^{-}(v)=k-1$, it follows from Proposition 17 (a) that there is a color $\alpha$ that appears in $N_{B}^{-}(v)$ but not in $N_{B}^{+}(v)$. Let $u$ be the vertex from $N_{B}^{-}(v)$ with $\varphi(u)=\alpha$. Note that Proposition 17(a) furthermore implies that there is a vertex in $v^{\prime} \in N_{D}^{+}(v) \cap(V(D) \backslash V(B))$ that has color $\alpha$. First we show that $d_{B}^{+}(v)=0$. Suppose, to the contrary, that $d_{B}^{+}(v)>0$ and let $w$ be an out-neighbor of $v$ in $D_{L}$. Then, in $B$ there is a (not-necessarily directed) induced cycle $C$ such that $u, v$ and $w$ are consecutive on $C$. Beginning with $u \rightarrow v$, we shift all vertices of $C$ clockwise and obtain a new $(k-1)$-coloring $\varphi^{\prime}$ of $D-v$ with $\varphi^{\prime}(w)=\alpha$. Since no vertex from $V(D) \backslash V(C)$ took part in the shifting, we have $\varphi^{\prime}\left(v^{\prime}\right)=\varphi\left(v^{\prime}\right)=\alpha$ and so $\alpha$ appears twice in $N_{D}^{+}(v)$, contradicting Proposition 17(a). This proves that $d_{B}^{+}(v)=0$. 
Let again $C$ be an (undirected) induced cycle in $B$ such that $u$ and $v$ are consecutive on $C$ and let $w$ be the other neighbor of $v$ in $C$. Then, $w$ is also an in-neighbor of $v\left(\operatorname{as~}_{B}^{+}(v)=\right.$ $0)$. Thus, it follows from Proposition 17 (a) that $\varphi(w) \neq \varphi(u)$, say $\varphi(w)=\beta$. Moreover, we obtain that the vertices of $C$ (except from $v$ ) are colored alternately with $\beta$ and $\alpha$. Otherwise, there are two consecutive vertices $x, x^{\prime}$ on $C$ such that $\left\{\varphi(x), \varphi\left(x^{\prime}\right)\right\} \neq\{\alpha, \beta\}$. Then we can shift the colors around the vertices of $C$ such that $u$ gets color $\varphi(x)$ and $w$ gets color $\varphi\left(x^{\prime}\right)$ and obtain a $(k-1)$-coloring $\varphi^{\prime}$ of $D-v$ with $\left\{\varphi^{\prime}(u), \varphi^{\prime}(w)\right\} \neq\{\alpha, \beta\}$, which contradicts Proposition 17(a) as $C$ is induced and so no neighbors of $v$ besides $u$ and $w$ take part in the shifting.

As a consequence, $C$ has odd length. Now let $v=v_{1}, w=v_{2}, v_{3}, \ldots, u=v_{r}, v_{1}$ be a cyclic ordering of the vertices of $C$. We claim that $v_{3} v_{2} \notin A(D)$. Assume, to the contrary, $v_{3} v_{2} \in A(D)$. Then, we can shift $w \rightarrow v$ and obtain a coloring $\varphi^{\prime}$ of $D-w$ with $\varphi^{\prime}(v)=\beta$ and $\varphi^{\prime}\left(v_{3}\right)=\alpha$. In particular, $v_{3}$ is the only in-neighbor of $w$ that has color $\alpha$ with respect to $\varphi^{\prime}$. On the other hand, beginning from $\varphi$ with $u \rightarrow v$, we can shift every vertex besides $v$ clockwise around $C$ (the last shift is $\left.w \rightarrow v_{3}\right)$ and get a $(k-1)$-coloring $\varphi^{*}$ of $D-w$ with $\varphi^{*}(v)=\alpha$ and $\varphi^{*}\left(v_{3}\right)=\beta$. As $v w \notin A(D)$ and as $C$ is induced, it follows that $w$ has no in-neighbor that has color $\alpha$ with respect to $\varphi^{*}$, a contradiction. Hence, $v_{3} v_{2} \notin A(D)$ and so $v_{2} v_{3} \in A(D)$. By repeating this argumentation, we obtain that $v_{i+1} v_{i} \notin A(D)$ but $v_{i} v_{i+1} \in A(D)$ for $i \geqslant 2$ even and that $v_{i} v_{i+1} \notin A(D)$ but $v_{i+1} v_{i} \in A(D)$ for $i \geqslant 3$ odd. In particular, this leads to $v_{r} v \notin A(D)$, a contradiction. This proves the claim.

Now let $\varphi$ be a $(k-1)$-coloring of $D-B$ with color set $\Gamma=\{1,2, \ldots, k-1\}$. For $v \in V(B)$, let

$$
L(v)=\Gamma \backslash \varphi\left(N_{D}^{+}(v) \backslash V(B)\right) .
$$

Then, as $d_{D}^{+}(v)=d_{D}^{-}(v)=k-1=|\Gamma|$ and since $d_{B}^{+}(v)=d_{B}^{-}(v)$ by Claim 1 , we have $|L(v)| \geqslant \max \left\{d_{B}^{+}(v), d_{B}^{-}(v)\right\}$ for all $v \in V(B)$. Moreover, $B$ is not $L$-colorable, as the union of any $L$-coloring of $B$ with $\varphi$ would clearly lead to a $(k-1)$-coloring of $D$. Hence, we can apply Theorem 16 and so $B$ is a directed cycle, or an odd bidirected cycle, or a bidirected complete graph, as claimed.

In the undirected case, Gallai [7] showed that the only blocks of the low vertex graph are complete graphs or odd cycles. Although for digraphs the directed cycles arise naturally, it may surprise that there can also be blocks that consist of just one arc. That this indeed may happen is illustrated in Figure 7, where we show the Hajós join of two copies of $D\left(K_{4}\right)$; here the low vertex subdigraph consists of every vertex except the identified vertex $v$. Clearly, by starting from $D\left(K_{k}\right)$ and iteratively taking Hajós joins with another copy of $D\left(K_{k}\right)$, we can even create infinite families of digraphs $D$ such that there are blocks of $D_{L}$ consisting of just a single arc.

Gallai used the characterization of the low vertex subgraph of critical graphs he obtained in [7] to establish a lower bound for the number of edges of critical graphs. We can apply the same approach to obtain a similar bound for the number of arcs in critical digraphs. 


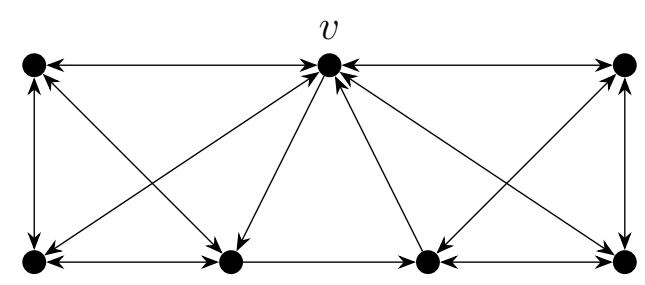

Figure 7: The Hajós join of two bidirected $K_{4}$.

Theorem 18. Let $D$ be a $(k+1)$-critical digraph with $k \geqslant 3$ and without digons. Then

$$
2|A(D)| \geqslant\left(2 k+\frac{k}{3 k+1}\right)|D|
$$

Proof. Let $V=V(D)$ and let $n=|V|$. For a set $X \subseteq V$, let $a(X)$ denote the number of $\operatorname{arcs}$ of $D[X]$. Furthermore, let

$$
R=\left(2 k+\frac{k}{3 k+1}\right)
$$

Our aim is to show that $2 a(V) \geqslant R n$. If $\left|D_{L}\right|=0$, then every vertex $v$ of $D$ satisfies $d_{D}^{+}(v)+d_{D}^{-}(v) \geqslant 2 k+1$, which leads to $2 a(V) \geqslant(2 k+1) n \geqslant R n$, and we are done. So assume that $\left|D_{L}\right| \geqslant 1$. Since $D$ has no digons, it follows from Theorem 15 that each block of $D_{L}$ consists of an isolated vertex, or exactly one arc, or is a directed cycle of length at least three.

Now we claim that $3\left|D_{L}\right| \geqslant 2\left|A\left(D_{L}\right)\right|$. If $D_{L}$ is not connected, then it suffices to prove this claim for each component of $D_{L}$. Thus, we may assume that $D_{L}$ is connected. The proof of the inequality is by induction on the number of blocks of $D_{L}$. If $D_{L}$ itself is a block, the statement clearly holds. If $D_{L}$ consists of more than one block, let $B$ be an end-block of $D_{L}$, i.e., $B$ is a block of $D_{L}$ containing exactly one separating vertex $v_{B}$ of $D_{L}$. Now let $D_{L}^{\prime}=D_{L}-\left(V(B) \backslash\left\{v_{B}\right\}\right)$. Then, by the induction hypothesis, we have $3\left|D_{L}^{\prime}\right| \geqslant 2\left|A\left(D_{L}^{\prime}\right)\right|$. As $B$ either consists of exactly one arc or is a directed cycle of length $\ell \geqslant 3$, we have $3(|B|-1)-2|A(B)| \geqslant 0$. This leads to

$$
3\left|D_{L}\right|=3\left|D_{L}^{\prime}\right|+3(|B|-1) \geqslant 2\left|A\left(D_{L}\right)\right|-2|A(B)|+3(|B|-1) \geqslant 2\left|A\left(D_{L}\right)\right|,
$$

which proves the claim.

Since every vertex of $U=V\left(D_{L}\right)$ has total degree $2 k$ in $D$ (i.e., $d_{D}^{+}(v)+d_{D}^{-}(v)=2 k$ for all $v \in U$ ) and since $k \geqslant 3$, we obtain that

$$
2 a(V)=2 a(W)+4 k|U|-2 a(U) \geqslant 4 k|U|-2 a(U) \geqslant(4 k-3)|U| \geqslant 3 k|U|
$$

On the other hand, since every vertex in $W$ has total degree at least $2 k+1$ and since $n=|U|+|W|$, we have

$$
2 a(V) \geqslant 2 k n+|W| \geqslant(2 k+1) n-|U|
$$


Adding the first inequality to the second inequality multiplied with $3 k$ yields

$$
2 a(V)(3 k+1) \geqslant 3 k(2 k+1) n,
$$

and, as $3 k(2 k+1)=2 k(3 k+1)+k$, we conclude

$$
2 a(V) \geqslant\left(2 k+\frac{k}{3 k+1}\right) n=R n .
$$

Thus, the proof is complete.

\section{Open Questions}

Since the field of critical digraphs is still wide open, a lot of questions immediately come to mind. It follows from Theorem 4 that each $k$-critical digraph is Hajós- $k$-constructible. However, the proof of Theorem 4 is not constructive at all and the authors feel quite embarrassed in admitting that they could not even manage to construct a bidirected cycle of length five from bidirected $K_{3}$ 's using directed Hajós joins and identification of non-adjacent vertices. Thus, we want to pose the following question.

Question 19. How can a bidirected $C_{5}$ be constructed from copies of $D\left(K_{3}\right)$ by only using directed Hajós joins and identifying non-adjacent vertices?

Building upon this question, it is of particular interest to study the connection of the Hajós construction to computational complexity. In the undirected case, Mansfield and Welsh [22] stated the problem of determining the complexity of the Hajós construction. They noted that if for any $k \geqslant 3$ there would exist a polynomial $P$ such that every graph of order $n$ with chromatic number $k$ contains a Hajós- $k$-constructible subgraph that can be obtained by at most $P(n)$ uses of the Hajós-join and identification of nonadjacent vertices, then NP $=$ coNP. Hence, it is very likely that the Hajós construction is not polynomially bounded, but not much progress has been made on this problem yet. Pitassi and Urquhart [30] found a linkage to another important open problem in logic; they proved that a restricted version of the Hajós construction is polynomially bounded if and only if extended Frege systems are polynomially bounded.

Question 20. For $k \geqslant 3$, is there a polynomial $P$ such that every digraph of order $n$ contains a Hajós- $k$-constructible subdigraph that can be obtained from bidirected complete graphs of order $k$ by at most $P(n)$ uses of the directed Hajós-join and identification of non-adjacent vertices?

A beautiful theorem of Gallai [8] states that any $k$-critical graph with order at most $2 k-2$ and $k \geqslant 2$ is the Dirac join of two disjoint non-empty critical graphs (for the Dirac join of two undirected graphs just add all possible edges between the two graphs $G_{1}$ and $G_{2}$ ). Within the last decades, various different proofs of this theorem have been published (see e.g. [25] and [31]). Clearly, a graph $G$ is the Dirac join of two disjoint non-empty graphs if and only if $\bar{G}$ is disconnected and so most of the proofs use matching theory for the complement graph $\bar{G}$. In a previous version of this paper we raised the following question. 
Question 21. Let $k \geqslant 3$ be an integer. Is there a $k$-critical digraph $D$ on at most $2 k-2$ vertices that is not the Dirac join of two proper digraphs $D_{1}$ and $D_{2}$ ?

The (negative) answer to this question was recently given by Stehlík [32].

Theorem 22 (Stehlík). Let $k \geqslant 3$ be an integer and let $D$ be a $k$-critical digraph on at most $2 k-2$ vertices. Then, $\bar{D}$ is disconnected and so $D$ is the Dirac join of two proper subdigraphs $D_{1}$ and $D_{2}$.

In coloring theory of digraphs, it is often of particular interest how digon-free digraphs behave. For example, it was shown by Harutyunyan in his $\mathrm{PhD}$ thesis [12] that almost all tournaments of order $n$ have dichromatic number at least $\frac{1}{2}\left(\frac{n}{\log n+1}\right)$. As a consequence, if $n$ is large enough, then for some $k \geqslant \frac{1}{2}\left(\frac{n}{\log n+1}\right)$ there are $k$-critical digon-free digraphs on at most $n$ vertices. This leads to our final question.

Question 23. For fixed $k \geqslant 3$, what is the minimum integer $N(k)$ such that there is a $k$-critical digon-free digraph on $N(k)$ vertices?

As $k-1 \leqslant \min \left\{d_{D}^{+}(v), d_{D}^{-}(v)\right\}$ for all vertices $v$ of a $k$-critical digraph $D$, we trivially have $N(k) \geqslant 2 k-1$. In fact, Brooks' theorem for digraphs [24] implies that $N(k) \geqslant 2 k$ for $k \geqslant 3$. Moreover, some small values are already known: the directed triangle shows that $N(2)=3$, and Neumann-Lara [28] proved that $N(3)=7, N(4)=11$, and $17 \leqslant N(5) \leqslant 19$; he conjectured that $N(5)=17$.

It follows from Theorem 18 that each 3-critical digon-free digraph $D$ satisfies $|A(D)| \geqslant$ $\left(2+\frac{1}{7}\right)|D|$. We believe that this bound can be improved to $|A(D)| \geqslant\left(2+\frac{1}{2}\right)|D|$. As a consequence of our bound, we obtain that if $D$ is a 3-critical digon-free planar digraph, then $G(D)$ contains a triangle. However, Li and Mohar [21] in fact proved that in this case $D$ contains a directed cycle of length three. This implies that every planar digraph of digirth at least 4 admits a 2-coloring. This result is a first step in proving the following, famous conjecture proposed by Erdös and Neumann-Lara, and, independently, by Škrekovski (for a reference see [21]).

Conjecture 24 (Neumann-Lara). Every digon-free planar digraph $D$ satisfies $\vec{\chi}(D) \leqslant 2$.

\section{Acknowledgements}

The authors would like to thank both referees for their valuable comments.

\section{References}

[1] S. D. Andres and W. Hochstättler. Perfect digraphs. J. Graph Theory 79 (2015) 21-29.

[2] J. Bang-Jensen and G. Gutin. Digraphs: Theory, Algorithms and Applications (Second Edition). Springer-Verlag London Limited, London 2009. 
[3] M. Chudnovsky, N. Robertson, P. Seymour, and R. Thomas. The strong perfect graph theorem. Ann. Math. 164 (2006) 51-229.

[4] R. P. Dilworth, A decomposition theorem for partially ordered sets. Ann. Math. 51 (1950) 161-166.

[5] G. A. Dirac. On the Colouring of Graphs. University of London, 1951.

[6] T. Gallai and A. N. Milgram. Verallgemeinerung eines graphentheoretischen Satzes von Rédei. Acta Sci. Math. Szeged 21 (1960) 181-186.

[7] T. Gallai. Kritische Graphen I. Publ. Math. Inst. Hungar. Acad. Sci. 8 (1963) 165192.

[8] T. Gallai. Kritische Graphen II. Publ. Math. Inst. Hungar. Acad. Sci. 8 (1963) 373395.

[9] S. Gravier. A Hajós-like theorem for list coloring. Discrete Math. 152 (1996) 299-302.

[10] G. Hajós. Über eine Konstruktion nicht n-färbbarer Graphen. Wiss. Z. Martin Luther Univ. Halle-Wittenberg, Math.-Natur. Reihe 10 (1961) 116-117.

[11] D. Hanson, G. C. Robinson, and B. Toft. Remarks on the graph colour theorem of Hajós. Cong. Numer. 55 (1986) 69-76.

[12] A. Harutyunyan. Brooks type results for coloring of digraphs. Simon Fraser University Vancouver, 2011.

[13] A. Harutyunyan and B. Mohar. Strengthened Brooks Theorem for digraphs of girth three. Electr. J. Combin. 18 (() 1.)2011\#P195

[14] A. Harutyunyan and B. Mohar. Gallai's theorem for list coloring of digraphs. SIAM J. Discrete Math. 25 (2011) 170-180.

[15] R. Hoshino and K. Kawarabayashi. The edge density of critical digraphs. Combinatorica 35 (2015) 619-631.

[16] T. R. Jensen. Grassmann homomorphism and Hajós type theorems. Linear Algebra Appl. 522 (2017) 140-152.

[17] T. R. Jensen and B. Toft. Graph Coloring Problems. Wiley-Interscience, New York 1995.

[18] Y. Kang. Hajós-like theorem for signed graphs. European J. Combin. 67 (2018) 199207.

[19] D. König. Über Graphen und ihre Anwendung auf Determinantentheorie und Mengenlehre. Math. Ann. 77 (1916) 453-465.

[20] D. Král. Hajós' theorem for list coloring. Discrete Math. 287 (2004) 161-163.

[21] Z. Li and B. Mohar. Planar digraphs of digirth four are 2-colorable. SIAM J. Discrete Math. 31 (2017) 2201-2205.

[22] A. J. Mansfield and D. J. A. Welsh. Some coloring problems and their complexity. Ann. Discrete Math. 13 (1982) 159-170.

[23] B. Mohar. Hajós theorem for coloring of edge weighted graphs. Combinatorica 25 (2004) 65-76. 
[24] B. Mohar. Eigenvalues and colorings of digraphs. Linear Algebra and its Applications 432 (2010) 2273-2277.

[25] M. Molloy. Chromatic neighborhood set. J. Graph Theory 31 (1999) 303-311.

[26] J. Nešetril. The homomorphism structure of classes of graphs. Combin. Probab. Comput. 8 (1999) 177-184.

[27] V. Neumann-Lara. The dichromatic number of a digraph. J. Combin. Theory Ser. B 33 (1982) 265-270.

[28] V. Neumann-Lara. The 3- and 4-chromatic tournaments of minimum order. Discrete Math. 135 (1994) 233-243.

[29] Ø. Ore. The Four Color Problem. Academic Press, New York 1967.

[30] T. Pitassi and A. Urquhart. The complexity of the Hajós calculus. SIAM J. Discrete Math. 8 (1995) 464-483.

[31] M. Stehlík. Critical graphs with connected complements. J. Combin. Theory Ser. B 89 (2003) 189-194.

[32] M. Stehlík. Critical digraphs with few vertices. arXiv: 1910.02454 (2019).

[33] A. Urquhart. The graph constructions of Hajós and Ore. J. Graph Theory 26 (1997) $211-215$.

[34] X. Zhu. An analogue of Hajós' theorem for circular chromatic number II. Graphs Combin. 265 (2003) 419-432. 